\title{
Enterocolopatía sprue-like asociada a la toma de olmesartán
}

\section{Olmesartan-induced sprue-like enteropathy}

\author{
César Arranz-Solana, Sara Pérez-Moyano, lorena Morillo-Blanco, \\ Julio Guilarte López-Mañas • Granada (España)
}

DOI: https://doi.org/10.36104/amc.2022.2203

\section{Resumen}

La diarrea crónica es una patología frecuente con un amplio diagnóstico diferencial. Presentamos el caso de un paciente con diarrea crónica secundaria a enterocolopatía por la toma prolongada de olmesartán. Se trata de una patología infradiagnosticada por desconocimiento de la entidad, pero que debe considerarse en base a la frecuencia de uso del grupo farmacológico. En nuestro caso, la buena evolución clínica tras la retirada del fármaco nos muestra la necesidad de considerarlo como causa directa del cuadro clínico. Un adecuado enfoque terapéutico en estos pacientes nos permitirá evitar pruebas complementarias, costos innecesarios y se traducirá en una mejora diagnóstica y del pronóstico de estos pacientes. (Acta Med Colomb 2022; 47.DOI: https://doi.org/10.36104/amc.2022.2203).

Palabras clave: olmesartán, enteropatía, sprue-like, diarrea crónica, pérdida de peso.

\section{Abstract}

Chronic diarrhea is a common pathology with a broad differential diagnosis. We present the case of a patient with chronic diarrhea secondary to enteropathy due to prolonged consumption of olmesartan. This is an underdiagnosed pathology due to lack of knowledge regarding this entity, but it should be considered, given the frequency with which this pharmacological group is used. In our case, the favorable clinical progression after withdrawing the medication indicates the need to consider it as the direct cause of the clinical picture. An appropriate therapeutic approach to these patients will allow us to avoid complementary tests and unnecessary costs, and will translate into a better diagnosis and prognosis in these patients. (Acta Med Colomb 2022; 47. DOI: https://doi. org/10.36104/amc.2022.2203).

Key words: olmesartan, enteropathy, sprue-like, chronic diarrhea, weight loss.
Dres. César Arranz-Solana, Sara Pérez-Moyano, Lorena Morillo-Blanco, Julio Guilarte LópezMañas: FEA UGC Medicina Interna, Especialidades Médicas y Cuidados Paliativos. Hospital Comarcal de Baza. Baza. Granada (España). Correspondencia: Dr. César Arranz-Solana. Granada (España).

E-Mail: cesar.arranz.sspa@juntadeandalucia.es Recibido: 11/V/2021 Aceptado: 31/VIII/2021

\section{Introducción}

El olmesartán es un antagonista del receptor tipo 1 de la angiotensina II utilizado habitualmente en el tratamiento de pacientes con hipertensión arterial. Recientemente se han descrito varios casos de enteropatía sprue-like asociados al uso de este fármaco tras meses o incluso años de comenzar el tratamiento (1). El síntoma más frecuente es la diarrea crónica grave con pérdida de peso y también pueden aparecer náuseas, vómitos y dolor abdominal. La deshidratación grave, insuficiencia renal aguda, hipoalbuminemia y anomalías electrolíticas pueden ser complicaciones de esta entidad, requiriendo ingreso hospitalario (2).

\section{Caso clínico}

Varón de 80 años con antecedentes de hipertensión arterial esencial y dislipemia de varios años de evolución.
En seguimiento por cardiología, por cardiopatía esclerohipertensiva, estenosis aórtica severa e insuficiencia aórtica moderada y desde hace más de cuatro años, con olmesartán medoxomilo/hidroclorotiazida 40/25 mg diarios como tratamiento antihipertensivo.

El paciente refiere desde hace cuatro meses cuadro diarreico de 10-15 deposiciones líquidas diarias sin claros productos patológicos. El cuadro clínico se acompañaba de una pérdida de $6 \mathrm{~kg}$ de peso en el último mes y medio. Previamente a acudir a urgencias de nuestro hospital había sido valorado en varias ocasiones por su médico de atención primaria, prescribiéndole finalmente ciprofloxacino y loperamida sin presentar mejoría. A su llegada a urgencias, presenta mareo inespecífico y sensación de enfermedad, destaca en la exploración física hipotensión arterial $(60 / 40 \mathrm{mmHg})$, palidez cutánea, signo del pliegue positivo, oligoanuria, 
todo ello en contexto de shock hipovolémico secundario a pérdidas digestivas, requiriendo tratamiento en observación de la unidad de urgencias. Analíticamente presentaba: úrea $127 \mathrm{mg} / \mathrm{dL}$, creatinina $10.91 \mathrm{mg} / \mathrm{dL}$, sodio $144 \mathrm{mEq} / \mathrm{L}$, potasio $2.90 \mathrm{mEq} / \mathrm{L}$ y proteína $\mathrm{C}$ reactiva $33.7 \mathrm{mg} / \mathrm{L}$. En la gasometría venosa destacaba un $\mathrm{pH}$ : 7.04 y bicarbonato $13.9 \mathrm{mmol} / \mathrm{L}$. Se realiza ecografía de abdomen de urgencias donde se visualizan los riñones de tamaño, ecoestructura y espesor parenquimatoso normal, descartándose además uropatía obstructiva. Una vez estabilizada su situación clínica se procede a un estudio exhaustivo, con los siguientes resultados: función hepática, vitamina B12, ácido fólico, hormonas tiroideas, cortisol basal, proteinograma sérico, coprocultivos, estudio de parásitos en heces, toxina Clostridioides difficile en heces, hemocultivos, anticuerpos antinucleares (ANA), anticuerpos anticitoplasma de neutrófilos (ANCA) y anticuerpos antitransglutaminasa IgA en rangos de la normalidad. Antes estos resultados se consulta caso con el servicio de digestivo, decidiendo realizar endoscopia digestiva alta e ileocolonoscopia. Tras la realización de las endoscopias, sólo destacaban múltiples divertículos en sigma, sin otras claras lesiones a nivel macroscópico. En el estudio de anatomía patológica de las biopsias realizadas mostraron en la segunda porción del duodeno: atrofia y acortamiento vellositario de distribución muy irregular con igualmente muy variable linfocitosis intraepitelial (entre 10 y 20 linfocitos por 100 enterocitos evaluada mediante CD3) sin apenas afectación del ápice vellositario, todo ello muy probablemente asociado a la ingesta crónica de olmesartán (Figura 1). A nivel colon, tras las múltiples biopsias realizadas se concluye como pancolitis linfoplasmocitaria moderada sugerentes de enterocolopatía sprue-like por olmesartán (Figura 2).

Ante la ausencia de criterios diagnósticos de enfermedad celiaca, tras los hallazgos de las biopsias y habiéndose descartado otras enfermedades orgánicas como causa del

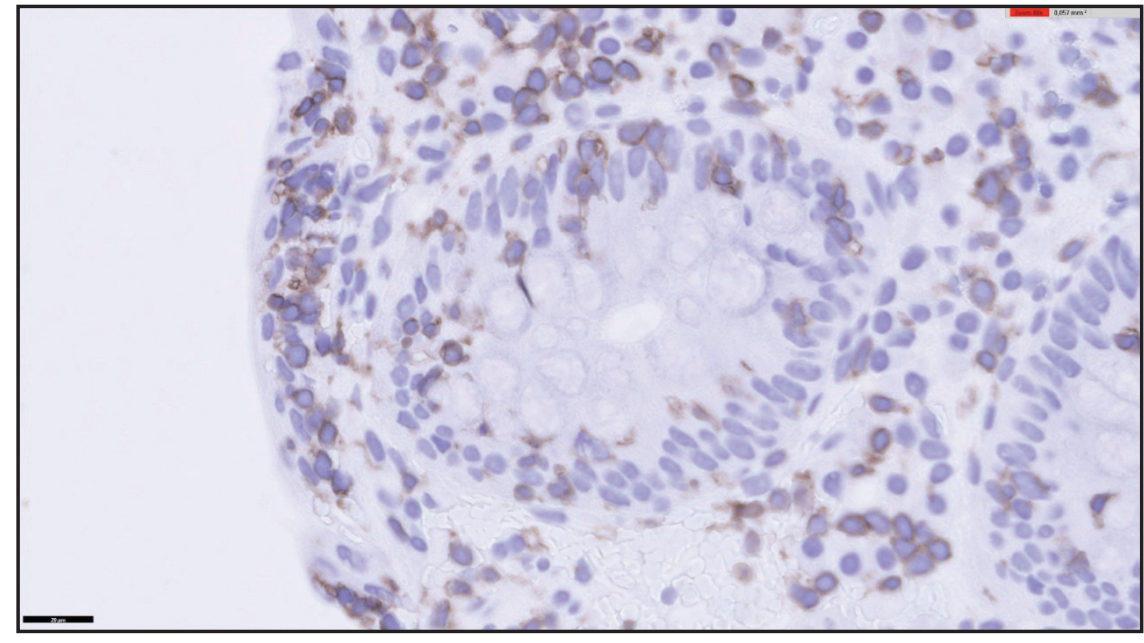

Figura 1. Biopsia duodenal. Inmunohistoquímica CD3, linfocitosis intraepitelial.

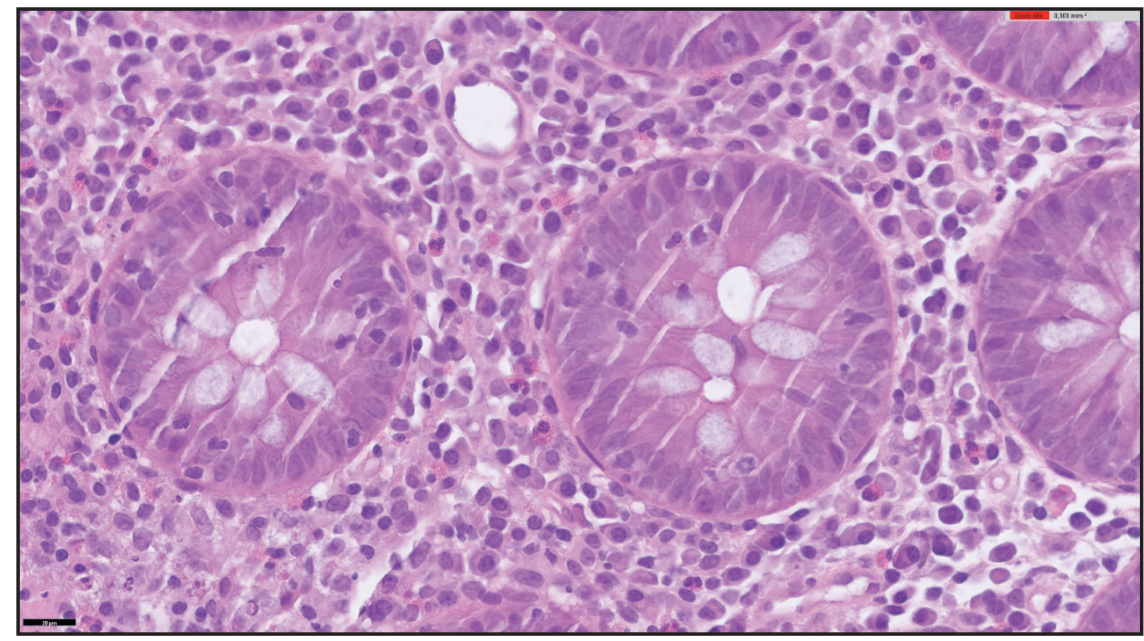

Figura 2. Biopsia colon. Hematoxilina-eosina $600 \mathrm{X}$, linfocitosis intraepitelial e infiltrado inflamatorio linfoplasmocitario. 
cuadro que presentaba el paciente, se consideró la toma de olmesartán como la causa más probable de la enterocolopatía, por lo que se suspendió la toma de dicho fármaco. Tras la supresión del olmesartán presentó resolución de la diarrea, recuperación ponderal y se mantuvo asintomático en controles posteriores.

\section{Discusión}

Rubio-Tapia y col. (3) describieron en 2012 la aparición de enteropatías sprue-like en estrecha relación con la toma de olmesartán, hecho que ha sido posteriormente reforzado por la agencia del medicamento de los Estados Unidos. La enteropatía asociada con otros sartanes parece ser muy rara (4).

El mecanismo causal de esta patología es desconocido en el momento actual (3). Debido a la fisiopatología de la enfermedad, la clínica no se produce inmediatamente después de la introducción del fármaco, sino que es necesaria la alteración del epitelio duodenal a lo largo de meses o incluso años $(1,5)$. Los cambios histológicos descritos en la enteropatía inducida por olmesartán pueden variar desde linfocitosis intraepitelial y proliferación linfocítica de la lámina propia a atrofia vellositaria marcada (4), hallazgos encontrados en las biopsias del paciente que presentamos. Se ha descrito en la literatura inhibición del factor de crecimiento beta por parte de los inhibidores del receptor de angiotensina II (6), un factor importante para mantener la homeostasis inmunitaria intestinal. Se desconoce si este mecanismo puede relacionarse con la aparición de la atrofia vellositaria duodenal que presentan estos pacientes. El tratamiento de esta patología incluye la suspensión del fármaco y en casos graves, los esteroides orales o intravenosos han atenuado los síntomas (7). En el caso que presentamos, se suspendió el fármaco y se inició dosis de esteroides a dosis bajas durante siete días, con mejoría progresiva del cuadro diarreico.

Por todo ello, consideramos necesario y más aún con el aumento de la prevalencia de dicha entidad en los últimos años, incluirla en el diagnóstico diferencial de síndromes diarreicos crónicos de etiología no aclarada (8). Dado que las complicaciones asociadas pueden ser potencialmente graves, es necesario en la historia clínica, un repaso exhaustivo del tratamiento farmacológico crónico del paciente.

\section{Referencias}

1. Esteve M, Termiño R, Carrasco A, Batista L, del Val A, Blé M, et al. Potential coeliac disease markers and autoimmunity in olmesartan induced enteropathy: A population based study. Dig Liver Dis. 2016;48:154-61.

2. Gonakoti S, Khullar S, Rajkumar A. Olmesartan associated enteropathy: a rare underdiagnosed cause of diarrhea and weight loss. Am J Case Rep 2019;20:111116.

3. Rubio-Tapia A, Herman ML, Ludvigsson JF, Kelly DG, Mangan TF, Wu TT et al. Severe spruelike enteropathy associated with olmesartan. Mayo Clin Proc 2012; 87:732-738.

4. Marthey L, Cadiot G, Seksik P, et al. Olmesartan-associated enteropathy: results of a national survey. Aliment Pharmacol Ther. 2014 Nov;40(9):1103-9.

5. De Gaetani M, Tennyson CA, Lebwohl B, et al. Villous atrophy and negative celiac serology: a diagnostic and therapeutic dilemma. Am J Gastroenterol. 2013;108:647-53

6. Kagami S, Border WA, Miller DE, Noble NA. Angiotensin II stimulates extracellular matrix protein synthesis through induction of transforming growth factor-beta expression in rat glomerular mesangial cells. J Clin Invest 1994;93:2431-7.

7. Sripriya Gonakoti, Sanjiv Khullar, Aarthi Rajkumar. Olmesartan Associated Enteropathy: A Rare Underdiagnosed Cause of Diarrhea and Weight Loss. Am J Case Rep. 2019; 20: 111-116

8. Solano-Iturri G, García-Jiménez N, Solano-Iturri J.D, Blanco-Sampascual S Enteropatía sprue-like asociada a olmesartán: causa emergente de diarrea crónica asociada a fármacos. Revista de Gastroenterología de México 2018;83:71-72. 